\title{
CIUDADANÍAS DIGITALES
}

PERSPECTIVAS DESDE LOS MEDIOS, EL PERIODISMO Y LA EDUCOMUNICACIÓN

Miguel Ezequiel Badillo Mendoza

Camila Pérez Lagos

Narcisa Jessenia Medranda Morales

María Isabel Cortés Cortés 


\section{Agradecimientos especiales}

a los gestores e integrantes del GT 19 Comunicación

digital, redes y procesos de ALAIC y a los investigadores

e investigadoras de diversas regiones del mundo

participantes en este libro, que con sus acciones indagan

exploran sueñan y construyen un mundo mejor a partir del

estudio de la relación entre tecnología y comunicación. 


\section{Primera SECCIÓN:}

El caso Cambridge Analytica en el periódico francés "Le Figaro":

enfoque pragmatista y discursivo del estudio de los acontecimientos

Camila Pérez Lagos

Medios digitales y ciberciudadanía ambiental

análisis del Blog "noalacolosahorrorosa"

Perspectivas desde los medios,
el periodismo y la educomunicación

ISBN: 978-958-5544-31-4

ISBN (Digital): 978-958-5544-32-1

\section{Editorial Politécnico Grancolombiano}

Calle 61 No. 7 - 66

Tel: 7455555 , Ext. 1516

Diciembre 2019

Editores

Miguel Ezequiel Badillo Mendoza

Narcisa Jez Lagos

María Isabel Cortés Cortés

Autores

Antonia Isabel Nogales-Bocio

Begoña Pérez Calle
Camila Pérez Lagos

Carmen Marta-Lazo

Carlos Eduardo Valderrama Higuera

Daniel Barredo Ibáñez

Garza Montemayor

Harvey Murcia Quiñones

Javier Gil Quintana

José Antonio Gabelas-Barroso

Juliana Colussi
Luis Cárcamo Ull

Luis Cárcamo Ulloa

Miguel A. Esteban-Navar

Miguel Ezequiel Badillo Mendoza

Narcisa Medranda Morales

Pilar Arranz Martínez

Sara Osuna-Acedo

Stefania Ramírez López

Victoria Dalila Palacios Mieles

Lider de publicaciones

Analista de Producción Editoria

Carlos Eduardo Daza Orozco

\section{Corrección de Estilo}

Diseño y Armada Electrónica

Diseño de portada

everry

Institución Universitaria Politécnico Grancolombian

Grupo de Investigación en Comunicación

e Información Digital (GICID).

Universidad Politécnica Salesiana del Ecua

Université Sorbonne Nouvelle - Paris 3

¿Cómo citar este libro?

Badillo Mendoza, M. E., Pérez Lagos, C., Medranda Morales, N. J. \& Cortés Cortés, M. I. (2019). CIUDADANIAS DIGITALES Perspectiva Bogotá: Institución Universitaria Politécnico Grancolombiano.

Creado en Colombia

Todos los derechos reservados

No se permite la reproducción total o parcial de esta obra, ni su incorporación a un sistema informático, ni su tratamiento en cualquier forma o medio existentes o por existr, sin el permiso previo y por escrito de la Edrico

Para usos académicos y cientificos, la Institución Universitaria Commons del contenido de la obra con: Atribución - No comercialSin derivar - Compartir igual

El contenido de esta publicación se puede citar o reproduci con propósitos académicos siempre y cuando se dé la fuente

Las opiniones expresadas son responsabilidad exclusiva del

La Editorial del Politécnico Grancolombiano pertenece a la
Asociación de Editoriales Universitarias de Colombia (ASEUC)
Carmen Marta - Lazo, Miguel Ezequiel Badillo Mendoza

Los pasos de la memoria en tiempos de la ciudadanía digital

Harvey Murcia, Jenny Juliana Jiménez Rodríguez

Comunicación transmedia y ciudad. El caso de La Candelaria de Bogotá, Colombia

Stefania Ramírez López

Periodismo digital e inclusión de comunidades diversas de la región Amazónica:

estudio del sitio web InfoAmazônia

Paula Melani Rocha Universidade Estadual de Ponta Grossa, Juliana Colussi, Universidad del Rosario, Abinoan Santiago

SEGUNDA SECCIÓN :

Medios sociales, capital social y sentimiento de eficacia política: Un estudio comparativo entre las juventudes de México y Perú ......................................................................................................... 133

Estrategias europeas contra el discurso del odio

Begoña Pérez Calle, María Gómez y Patiño, Miguel Ángel Esteban-Navarro

De la infancia consumidora a la adolescencia creadora. Competencias productivas transmedia para la Cibersociedad

Javier Gil Quintana, Sara Osuna-Acedo

Una mirada a la sociedad de la transparencia en Latinoamérica Narcisa Jessenia Medranda Morales, Victoria Dalila Palacios Mieles

\section{TERCERA SECCIÓN}

Competencias digitales para la docencia no presencial. Protocolo de seguimiento, evaluación y pautas de tutorización online

Antonia Isabel Nogales-Bocio, José Antonio Gabelas-Barroso, Pilar Arranz Martínez

CiudadaniaSUR: Comunicación educativa para la formación ciudadana en el sur de Chile ...... 229 Luis Cárcamo Ulloa 


\section{Prólogo}

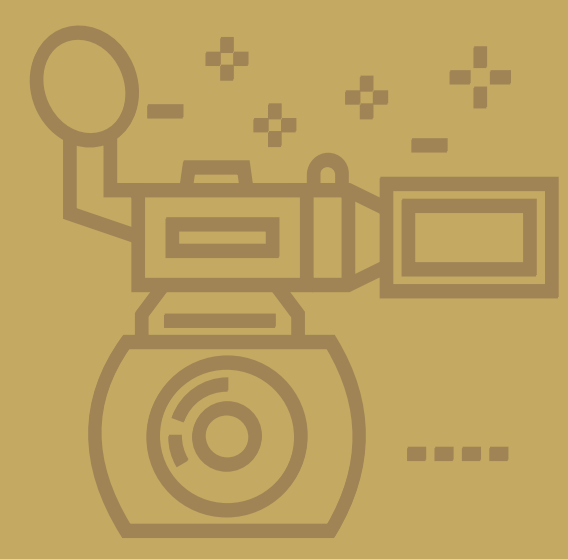

n cada época se dan unos cambios sociales fruto de las transformaciones tecnológicas. En la era actual, las Tecnologías de la Relación, la Información y la Comunicación (TRIC) (Marta-Lazo \& Gabelas, 2016) han generado un sinfín de posibilidades de interacción, en las que el Factor Relacional es el protagonista. Esto facilita que la ciberciudadanía pueda llevar a cabo procesos de intercambio de información hasta hace unas décadas impensables, en los que el espacio y el tiempo minimizan sus dimensiones, siendo la inmediatez y la ubicuidad dos de los grandes beneficios. El ejercicio de muchos de los derechos ciudadanos hoy ha trascendido a la Red.

Este libro trata de dar respuesta, entre otros objetivos, al papel de la ciberdemocracia participativa -a través del derecho a expresar opiniones o a decidir y reivindicar determinados temas que afectan lo cotidiano- como garantía de una libertad con mayor potencial. De esta manera, se plantea cómo el candelero político ha trasvasado los hemiciclos, donde la nueva ágora digital es el terreno de juego en el que ya todas y todos pueden decidir qué acciones realizar y qué actividades proponer, tomando en consideración también que existen intereses que van más allá de la ciudadanía.

La Red ha evolucionado desde un estado de entorno de referencia, a uno nuevo de pertenencia. La mediamorfosis (Fidler, 1997) ha convertido las distintas 
extensiones de la Web 2.0 en un flujo de inmersiones e interacciones, en las cuales las simulaciones adquieren una preponderancia especial. Hoy en día no solo se convive "con" la social media sino que se vive "en" estos entornos, involucrándonos de manera directa en un story telling donde cada uno somos protagonistas de nuestras vivencias, con una narrativa continuada. Los ciudadanos y ciudadanas somos artífices de nuestro "yo" virtual y de los roles que en cada momento asumimos. El nuevo modelo ecológico de la comunicación (Bronfenbrenner, 1977) pasa por la transformación de los exosistemas mediáticos a conformarse como agentes primarios dentro de los micro y mesosistemas, sirviendo de manera transversal para establecer relaciones con el resto de las personas e instituciones cercanas con las que convivimos.

En el universo digital se dan cita todo tipo de intercambios, experiencias e informaciones. Ante la gran amalgama de mensajes, nos planteamos cómo debe prepararse la ciudadanía para llevar a cabo interacciones en un sentido cívico, crítico y saludable. La vorágine de informaciones, muchas veces contaminadas, hace necesario que los interactuantes (Castells, 1998; Osuna Acedo, 2010; Osuna-Acedo, 2017) deban saber cómo filtrar la información, cómo contrastar fuentes y cómo distinguir entre información veraz y noticias falsas.

Las diferentes opciones con las que cuentan hoy los EMIRECs (emisoresreceptores, Cloutier, 1973) para ofrecer o recibir información de manera indistinta requieren que deban tomar conciencia de que sus actuaciones pueden convertirse en un arma de fuego en algunos casos y que se deben explotar todas las posibilidades que tienen Internet y las redes sociales, pero siempre desde un uso positivo. La recuperación de los valores sociales es algo que debemos exigirnos: la empatía, el respeto mutuo, la escucha activa, la interacción sana, la denuncia de lo nefasto y así un largo etcétera de aspectos éticos que conjuntan con las claves de cómo actuar en un entorno digital, a modo de formas de actuación en los escenarios presenciales. Desde este prisma, pensamos que hay que recuperar el fundamento del sentido del hombre y la mujer de manera prioritaria, por delante de la tecnología. De hecho, los nuevos equipamientos, dispositivos en su mayoría móviles, están al servicio de la ciudadanía. Así, llegamos a la nueva dimensión del humanismo digital.

Desde la trascendencia del diálogo y de la puesta en común, la obra que tengo el gusto de presentarles se inicia con un conversatorio llevado a cabo en el contexto de la VII Cátedra de Comunicación UNAD, con el título "Ciudadanías digitales" en el que se dan cita varias voces autorizadas en la materia (Miguel Ezequiel Badillo Mendoza, Carlos Valderrama, Camila Pérez Lagos, Juliana Jiménez, Harvey Murcia), quienes debaten sobre temas tan nucleares como la participación, la identidad, la seguridad o la vigilancia digital en las prácticas y movimientos de ciberciudadanía.

Tras esta introducción, a modo de conversatorio, la obra se estructura en tres secciones. La primera introduce un panorama contextual relativo al periodismo digital en el siglo XXI, mediante distintos estudios de caso, a lo largo de un total de 5 capítulos. En el primero, Camila Pérez Lagos presenta el caso Cambridge Analytica en el periódico francés "Le Figaro", con un enfoque pragmatista y discursivo del estudio de los acontecimientos. En el siguiente, Carmen Marta-Lazo y Miguel Ezequiel Badillo Mendoza realizan un estudio de los medios digitales y ciberciudadanía ambiental, por medio del análisis del Blog "noalacolosahorrorosa". El capítulo 3 plantea los pasos de la memoria en tiempos de la ciudadanía digital desde la mirada de Harvey Murcia y Jenny Juliana Jiménez. A continuación, Stefania Ramírez López se adentra en las relaciones entre comunicación transmedia y ciudad, mediante el estudio del caso de La Candelaria de Bogotá, Colombia. Cierra esta sección el capítulo firmado por Paula Melani Rocha, Juliana Colussi y Abinoan Santiago, en el que analizan el periodismo digital y la inclusión de comunidades diversas de la región Amazónica, mediante el estudio de InfoAmazonia.

La segunda sección trata la traslación de los medios a la ciudadanía digital, a lo largo de cuatro capítulos. La primera de las contribuciones se refiere a los medios sociales, capital social y sentimiento de eficacia política, a través de un estudio comparativo entre las juventudes de México y Perú, elaborado de la mano de Daniel Javier de la Garza Montemayor, Amaro la Rosa, Daniel Barredo Ibáñez y Úrsula FreundtThurne. La siguiente, contempla las estrategias europeas contra el discurso del odio, con un pormenorizado análisis llevado a cabo por Begoña Pérez Calle, María Gómez y Patiño y Miguel Esteban-Navarro. El tercer capítulo lleva por título "De la infancia consumidora a la adolescencia creadora. Competencias productivas transmedia para la Cibersociedad", con las firmas de Javier Gil-Quintana y Sara Osuna-Acedo. Para finalizar esta sección, Narcisa Jessenia Medranda Morales y Victoria Dalila Palacios Mieles destinan su espacio a ofrecer una mirada a la sociedad de la transparencia en Latinoamérica.

El libro se cierra con la tercera sección, destinada a la Educomunicación en la era global, que consta de dos capítulos. El primero se centra en las competencias digitales para la docencia no presencial y plantea un protocolo de seguimiento, evaluación y pautas de tutorización online, firmado por Antonia Isabel Nogales-Bocio, José Antonio Gabelas-Barroso y Pilar Arranz-Martínez. Y el último aborda la CiudadaniaSUR: Comunicación educativa para la formación ciudadana en el sur de Chile, escrito por Luis Cárcamo Ulloa. 
Con la conformación explicada previamente, esta publicación presenta un horizonte variado, con contextos y coyunturas distintas. Se trata de una obra poliédrica en cuanto a escenarios y estudios de caso, pero sin perder la esencia de una misma pieza angular, el estudio de la ciudadanía digital. En definitiva, es un libro que ofrece respuestas a las necesidades, demandas y acciones sociales que desde la Red de redes se generan y acontecen en ese intercambio de flujos informativos constantes de las TRIC.

Carmen Marta-Lazo

Profesora Titular

de la Universidad de Zaragoza (España)

\section{Referencias}

Bronfenbrenner, U. (1977): Toward an experimental ecology of human development. American Psychologist, 32, 513-531.

Castells, M. (1998): La era de la información: Economía, sociedad y cultura. La sociedad red. Vol. 1 Madrid: Alianza Editorial.

Cloutier, J. (1973): Le communication audio.scripto-visuelle á l'heure desd self-media. Canadá: Les Presses de I'Université de Montreal.

Fidler, R. (1997): Mediamorphosis. Understanding new media. Thousand Oaks, CA: Pine Forge Press.

Marta-Lazo, C.; Gabelas Barroso, J. A. (2016): Comunicación Digital. Un modelo basado en el Factor R-elacional. Barcelona: Editorial UOC.

Osuna Acedo, S. (2017): Gamificación y empoderamiento de los estudiantes en los SMOOC. El caso del proyecto europeo ECO. En P. Ávila Muñoz y C. Rama Vitale, Internet y Educación: amores y desamores. México: INFOTEC.

\section{Introducción} sincretismos y cotidianidad, el Grupo de Trabajo № 19, “Comunicación digital, redes y proceso" de la Asociación Latinoamericana de Investigadores en Comunicación (ALAIC) desarrolló un conversatorio sobre el tema ciudadanía(s) digital(es) el día 10 de octubre del 2018. Participaron los docentes e investigadores: Camila Pérez Lagos, de la Université Sorbonne Nouvelle-Paris 3, Carlos Eduardo Valderrama Higuera, de la Universidad Central, Harvey Murcia y Jenny Juliana Jiménez Rodríguez, del Politécnico Grancolombiano y Miguel E. Badillo Mendoza, de la UNAD. El mismo ejercicio se convirtió en un escenario de ciudadanía digital, sobre el cual se ampliaron las participaciones a otros investigadores que desde sus vertientes interpelan y marcan sentidos sobre la temática. El conversatorio giró sobre tres interrogantes: ¿Qué considera usted que son la/s ciudadanía(s) digital(es)?, ¿Qué tipo de relaciones se dan en la comunicación?, y ¿Desde su área de desempeño cómo lo evidencia? A continuación, se presenta la dinámica del evento, la cual mantiene los trazos de la oralidad para conservar las opiniones e ideas de los participantes:

\section{¿QUÉ CONSIDERA USTED QUE SON LA/S CIUDADANÍA(S) DIGITAL(ES)?}

Camila Pérez Lagos: "Buenas tardes, soy chilena, pero estoy trabajando en París ya hace algunos años. Hice mi tesis doctoral en Ciencias de la Información y de la Comunicación 
en la Universidad Sorbonne Nouvelle-Paris 3. Actualmente, mis intereses de investigación abarcan temas como el de la vigilancia digital, que es el tercer punto de las preguntas sobre el que me gustaría tomarme un poco más de tiempo. La definición de Ciudadanía es una palabra, que muchos autores coinciden en que permite profundizar diferentes significados. En primer lugar, designa una comunidad, un conjunto de individuos que están reunidos por el interés por la vida de la ciudad, el lugar en el que viven, que está enmarcado en convenciones, derechos, deberes y también en valores comunes. Pero también define una práctica, que es el enfoque que a mí me parece más interesante, ya que el ciudadano está invitado a participar en la vida democrática del entorno en el cual está inmerso, de hecho, jurídicamente los ciudadanos son individuos con derechos y deberes como lo dije anteriormente, como por ejemplo los deberes civiles, como pagar impuestos; en todos nuestros países es un hecho que debemos hacerlo, además de derechos civiles, derechos políticos, como el derecho (y el deber para algunos) de ir a votar, y también sociales, por ejemplo en Francia, algo que es muy común es el derecho social de hacer una huelga, el derecho social de tener una salud, el derecho social de tener una educación gratuita y de calidad, lo cual es normal aquí, pero que ocurre de manera más precaria en otros países como en el caso de Chile.

Para que el sujeto pueda adquirir y hacer práctica esta condición de "ciudadano", necesita informarse, debatir, criticar, votar y en algunos casos incluso, comprometerse con acciones colectivas, y eso es lo que entenderíamos por el hecho de ser ciudadano: constituye diferentes y variadas formas de actuar, desde votar hasta hacer una huelga o manifestarse. Junto con lo anterior, estas formas están vinculadas con los medios de comunicación. Dicho de otro modo, en los medios de comunicación es donde se visibiliza, donde se discuten los productos simbólicos como la información, los saberes y en general las ideologías, que igual integran esta participación ciudadana. En este sentido, para la ciudadanía, los medios son una herramienta de organización pública en relación con lo político, es decir con la discusión política que se pueda ejercer.

Como sabemos, con la llegada de las nuevas tecnologías de internet, se habla de una o de las ciudadanías digitales en plural. Para algunos, se definen como la capacidad de participar de la sociedad en línea, es decir los ciudadanos digitales serían quienes hacen uso de esta tecnología digital. Sin embargo, para otros, la ciudadanía digital incluye unas características potenciales democráticas, participativas, deliberativas, autónomas, que son prescritas legalmente y que están en perpetua negociación. El ejercicio de la ciudadanía digital implica la capacidad de vincularse con estos medios, que no es adquirida solamente por el hecho de estar en ese entorno o utilizarlas a diario; al contrario, implican una capacidad que necesita desarrollo. Por ejemplo, todos sabemos leer y escribir en línea, todos sabemos utilizar las redes sociales, nuestros estudiantes lo saben, sin embargo, es distinta la capacidad de relacionarse de una manera crítica con el entorno digital, para ello los ciudadanos necesitan adquirir unas competencias específicas. En este sentido, el ciudadano digital no es un usuario, es el portador de un proceso de apropiación, es decir, las personas se apropian de los medios para escucharse, reconocerse, un reconocimiento que no es pasivo, sino transformador Por ejemplo, en el caso de las organizaciones comunitarias donde las personas le dan sentido de pertenencia a las herramientas, en este caso digitales, las valora y aprenden a usarlas para satisfacer sus necesidades personales o los de un grupo social".

Miguel Badillo Ezequiel Mendoza: “Camila nos está remitiendo a algunos aspectos jurídicos, las prácticas, la ciudadanía, el derecho a la información, la importancia de la información como parte de un ejercicio efectivo de ciudadanía. Continuamos con el segundo participante, el profesor Carlos Valderrama, a quien le agradeceríamos que nos contara un poco de su perfil, e iniciamos con la primera pregunta".

Carlos Valderrama Higuera: "Muy buenas tardes, primero, quiero agradecer a la UNAD por la invitación; lo segundo, quiero felicitarlos por la iniciativa de esta publicación y de este nuevo escenario para pensar este tema, porque me parece que en Colombia ha faltado mucho más trabajo para dilucidar estos nuevos escenarios del ejercicio de la política. Yo soy Carlos Eduardo Valderrama, sociólogo de formación en pregrado, tengo una maestría en Sociología de la cultura y soy estudiante de doctorado en Comunicación con la Universidad Nacional de la Plata, trabajo con la Universidad Central, fundamentalmente en el tema de comunicación y educación.

Para entrar en materia, con respecto a la primera pregunta, quiero comenzar llamando la atención sobre un asunto que, desde hace ya varias décadas y desde distintos territorios se ha señalado, y es el hecho de que la política tradicional, la política en el sentido moderno, ha venido sufriendo una serie de transformaciones. Las nociones de política, las prácticas mismas de la cultura política y de la ciudadanía, han estado crecientemente interpeladas desde diversos lugares, básicamente por la promesa incumplida de la modernidad en relación con la construcción de una sociedad realmente democrática, pero también con la emergencia de nuevos escenarios tecnológicos y comunicativos, lo cual ha favorecido en gran medida, esos procesos de resignificación de la política y de lo político.

En ese marco, la noción de ciudadanía ya no se reduce al conjunto de derechos y deberes, como lo constituyó la modernidad; ya muchos autores, por mencionar algunos de Europa, Ulrich Beck y Giddens, en América Latina, Arturo Escobar, el mismo Norbert Lechner, el mismo Martin Hopenhayn, Pablo Salvat, se me escapan por su puesto muchos autores, han señalado que hoy lo que podemos entender es la ciudadanía 
más como una práctica, en contraposición a lo que se ha entendido como un estatus adquirido circunscrito a una serie de derechos y deberes. Prácticas inscritas en la vida cotidiana, como señalaban Arturo Escobar y Martin Hopenhayn, donde la cultura de la vida cotidiana se vuelve política, los hechos privados de la vida cotidiana e inscritos en la cultura se vuelven políticos, donde la participación no necesariamente pasa por las instancias tradicionales y las instituciones del Estado, en la que la práctica ciudadana y la expresión tienen un lugar fundamental.

Visto este panorama, entonces, con relación al tema de las ciudadanías digitales, no se pueden reducir a más de lo mismo: a reivindicar el derecho a tener internet, la conexión, al acceso a la información, esto es cierto, las ciudadanías digitales pasan por ahí, pero lo que yo planteo es que no se pueden agotar en ello, porque entre otras cosas, pensando que lo digital tiene continuidad con lo presencial, que hay una continuidad y una contigüidad entre esos dos escenarios sociológicos y políticos, el derecho a la expresión, el derecho a la comunicación, el derecho a la información, son exactamente los mismos en uno y otro espacio, y tampoco puede reducirse, como ha sido entendido por algunos sectores, al tema de gobierno electrónico, que las ciudadanías sean sinónimo de eficacia y eficiencia en la gestión de lo público, creo que estos son elementos que hay que trascender a la hora de pensar las ciudadanías digitales.

El ciberespacio es una ampliación del espacio político, justamente para desarrollar esas prácticas ciudadanas. Ejercer la ciudadanía en tanto práctica, como acción política participativa y de expresión, no puede entenderse como la última etapa o una etapa superior, es más bien una ampliación de ese espacio de lo público-político en el cual se da la construcción de sentido, en el cual se da la lucha por la significación política del ejercicio de la vida cotidiana".

Miguel Ezequiel Badillo Mendoza: "Perfecto profesor Carlos, vamos con la colega Juliana Jiménez, para que por favor responda a la primera pregunta, y luego Harvey Murcia continuaría”.

Juliana Jiménez Rodríguez: "Buenas tardes a los colegas y a los participantes, mi nombre es Juliana Jiménez, soy docente del Politécnico Grancolombiano, profesional en Estudios Literarios con maestría en Comunicación. Los colegas han abordado bastantes aspectos y han profundizado en el concepto de ciudadanías digitales y en la mayoría de los puntos que tocan estoy de acuerdo, recalcaría lo que mencionaba el profesor Carlos, de entender la ciudadanía digital desde las prácticas. Agregaría que más que una sola práctica, es un conjunto de prácticas que no necesariamente se construyen un ciento por ciento en el entorno digital, pensaría que son prácticas de naturaleza mixta, entonces algunas de estas prácticas son parcialmente digitales, otras prácticas serian ciento por ciento digitales, y entendiéndolo desde un aspecto político bastante amplio, como el profesor lo mencionaba, estas prácticas van a permear diferentes ámbitos, son prácticas que tienen que ver tanto con lo público como con lo privado, y permean la educación, la familia, la cultura, diferentes ambientes, y en esa medida creo que la ciudadanía digital tiene que ver tanto con las posibilidades de acceso y de brecha de lo digital, el tema de los derechos, y por supuesto uno de los temas fundamentales: el desarrollo de competencias para poder actuar y transitar en relación con el entorno digital".

Harvey Murcia: “Agradezco este encuentro tan fructífero, en aras de ir consolidando maneras de comprender estos nuevos escenarios que se están construyendo en la arena de la comunicación. Soy Semiólogo, especialista en Televisión y Magister en Comunicación, dirijo el Departamento de Medios Audiovisuales, y algunos posgrados relacionados con el área digital.

Sobre este tema, estamos de acuerdo en que tratar de comprender lo digital, o comprender las ciudadanías digitales es en primera instancia empezar a abandonar viejas categorías, que han perdido uso precisamente por las nuevas prácticas que se están dando en la arena cultural. Sobre ello, abordaría dos o tres dimensiones que nos permitirían aportar al debate que se está construyendo en este momento. En una primera instancia y siguiendo a Hanna Arent, tenemos que comprender esa discusión que hay entre Vita activa y Vita contemplativa, para entender esos rasgos de la modernidad como bien abordaba Carlos Valderrama, y sus efectos sobre la posmodernidad; no verlos como causas y efectos, sino verlos como esos proyectos fallidos que se han venido dando y que de una u otra manera han configurado otras maneras de comprender el entorno social. Desde allí, las ciudadanías digitales se comprenden por unos aspectos éticos, estéticos, políticos y económicos, que se evidencian en perspectivas tanto ontológicas como performativas, que atraviesan no solo las prácticas sociales, sino las subjetividades y las formas de habitar el mundo.

En este orden de ideas, yo creería que las ciudadanías digitales empiezan a tener un rasgo que rompe con el proyecto de modernidad, y es poner en la escena pública lo que es privado, y esto es lo que está marcando una corriente fundamental para entender las ciudadanías digitales, no tiene que ver con lo político, comprendido solamente como esa demanda de derechos y deberes, sino con empezar a comprender que hay otro tipo de sensibilidades que se van demostrando en prácticas que nos permiten evidenciar otras colectividades, otras formas de derechos, y otras subjetividades".

Miguel Ezequiel Badillo Mendoza: "Agregar algo después de cinco o seis personas que han dicho cosas tan importantes es un poco complejo; básicamente, he venido 
haciendo un rastreo de lo que es ese concepto y un poco entran varias situaciones que son históricamente importantes. Casi que los colegas están planteando lo básico, en dónde se enmarca la ciudadanía digital en esa crisis del concepto de ciudadanía, lo que se conecta con lo que nos dice el colega Harvey de esas nuevas subjetividades, donde la práctica de la ciudadanía ya no es tanto de lo político-jurídico, sino de aspectos más socioculturales, lo que nos permite encontrar lo que llaman derechos de tercera generación, donde a la persona le puede interesar y puede comprometerse más con una causa que está al otro lado del mundo y no interesarle lo que está pasando en su propia calle, o una serie de temáticas como la ecología, la economía, el género, donde de una u otra forma se ejerce ese proceso de la ciudadanía.

Se ha identificado la importancia de asumir una ciudadanía digital, para desde allí resaltar una ciudadanía vista desde una democracia representativa, ver los beneficios que las tecnologías nos permiten de cara al ejercicio de prácticas que incluso tienen un elemento político importante, porque casi que surgen desde el tema del gobierno electrónico, instaurado en el gobierno de Bill Clinton que es donde se empieza hablar de estos temas.

Creo que allí vamos hilando ideas, estamos hablando de subjetividades, estamos hablando de lo mixto que también es otro aspecto importante, y que el mismo Arturo Escobar lo plantea, el ciudadano digital es aquel que tiene la capacidad de estar en el escenario digital, en el ciberespacio y tener de la capacidad de actuar en política situada, él plantea que los problemas de la ciudadanía pueden estar en el ciberespacio pero tienen una incidencia y una raíz en hechos materiales o físicos".

Carlos Valderrama Higuera: "Un minutico para abordar algo que nos quedó faltando, primero quiero manifestar que me encuentro completamente de acuerdo con la profesora Juliana cuando hace la precisión en relación con las prácticas en el ejercicio de la ciudadanía, que son efectivamente prácticas mixtas, porque justamente hay una relación de continuidad y de contigüidad entre el espacio virtual y el presencial, y eso justamente me lleva a un punto que es clave, no solo por el hecho de que existen esos nuevos ecosistemas, sino que allí también se encuentran las hegemonías y los ejercicios modernos de la política.

En los escenarios virtuales y en los escenarios de la vida presencial que están atravesados y mediados por las tecnologías digitales de la información y la comunicación, se despliegan ejercicios autoritarios y hegemónicos de la política y de lo político, por eso es clave entender que las disputas por el poder, por la construcción del significado de la política, transitan desde lo presencial a lo virtual; en lo virtual hay unas disputas también muy potentes y significativas en relación con las utopías políticas, en relación con el sentido de sociedad, el sentido de ciudadanía, el sentido de participación".

\section{¿Qué tipo de relaciones se dan en la comunicación?}

Miguel Ezequiel Badillo Mendoza: "Entonces, continuando con nuestro conversatorio, en este tipo de escenario que acabamos de plantear, ¿qué tipo de relaciones se dan con la comunicación?"

Camila Pérez Lagos: "Lo primero que quiero señalar es que Internet en un principio se entendía como una opción o una posibilidad frente a los medios llamados tradicionales, hegemónicos, y que eran monopolios en algunos casos. Frente a los canales de visibilidad y el monopolio de algunos medios oficiales, los movimientos sociales se fueron apropiando de las redes sociales y de Internet para romper con el llamado "cerco mediático"; Internet apareció, en un principio, como un espacio de comunicación descentralizado, horizontal, más democrático, etc. Algunos ejemplos de lo anterior se evidencian en 2011 en Túnez, cuando los videos de violencia policial en la calle (viralizados en internet) hicieron que la movilización se organizara y sobretodo se viralizara en los canales de internet. Más recientemente, el uso del \#niunamenos, en el caso de América Latina, \#metoo en Estados Unidos, \#balancetonporc en Francia, fueron usados para visibilizar la violencia y el acoso hacia las mujeres.

Hay que hacer un alcance a lo dicho por los profesores, porque quiero reiterarlo. Ricardo Cardoso en 2012 plantea que el paisaje mediático actual no está necesariamente dominado por Internet, no es que haya aparecido y otros medios se silencien, sin embargo, está constituido por la superposición o la articulación de diferentes medios, no hay una separación binaria, no hay una participación "online" y "offline", pero hay una mixtura de diferentes tipos de medios.

En resumen, podemos decir que las tecnologías de internet permiten la creación de espacios de construcción y definición de grupos ciudadanos, y que a través de la puesta en escena de la circulación del relato y de los argumentos en las redes sociales, los ciudadanos comparten las injusticias sociales y los problemas políticos en general y eso es lo que hemos visto en los ejemplos que se citaron.

En relación con la tercera pregunta voy a hablar un poco del "lado oscuro" de la tecnología y sobre cómo las hegemonías se apropian de las redes sociales y de internet en general, como es el caso de las GAFAM". 
Carlos Valderrama Higuera: “Con relación a la segunda pregunta, quiero recordar cuatro elementos claves que en alguna oportunidad mencioné en alguno de mis textos: el componente comunicativo de la ciudadanía, brevemente para recordar, por una parte, el componente dialógico, que desde la antigüedad, desde la misma noción de ciudadanía de los antiguos es fundamental. Desde las más diversas orillas de la filosofía política, pasando por la teoría de la acción comunicativa, el construccionismo y la pedagogía crítica, se ha resaltado el componente dialógico, la dimensión argumentativa como algo fundamental del ejercicio ciudadano, y mucho más si la entendemos como una práctica, como lo hemos estado planteando.

El segundo componente es el componente narrativo, en el sentido de que para hacer una práctica ciudadana es necesario poder narrar como individuos, pero también como colectivo, como comunidad, poder narrar nuestras identidades, nuestra historia y nuestras memorias.

El otro componente que me parece clave es el componente hermenéutico, poder leer el contexto, los territorios físicos y virtuales, esos espacios desde donde actuamos políticamente, desde donde ejercemos la ciudadanía.

Y el último componente comunicativo es lo que llamaríamos el componente tecno-comunicativo o mediático, y ya lo habían planteado por ahí, es saber hacer todo el ejercicio, todo lo que le compete al ejercicio y a la práctica ciudadana con los viejos y nuevos escenarios y los repertorios tecnológicos en esas prácticas de comunicación.

Entendido esto y retomando lo que habíamos planteado, que las tecnologías digitales de la información y la comunicación han amplificado los escenarios de las prácticas de la ciudadanía, pues esas tecnologías han amplificado también y han permitido una especie de diáspora de las prácticas de la comunicación política, y han permitido a su vez generar nuevas prácticas, una emergencia de otras maneras de comunicación política; lo cual es evidente, así unos colectivos y unos sectores de la sociedad insistan y persistan en seguir con las prácticas modernas, unidireccionales, verticales e informacionistas de la comunicación.

En ese sentido, desde el punto de vista comunicativo, insisto en que la dimensión de la comunicación política hoy como nunca tiene un escenario ampliado, el espacio de la lucha por el sentido de lo político; es una esfera ampliada que permite muchos escenarios para la participación, para el ejercicio del poder, para la expresión narrativa, permitiendo poder narrarnos como pueblo, como comunidad y como sociedad.
Estas tecnologías han permitido la incorporación y convergencia de distintos lenguajes, no porque ellos no existieran, sino porque han potenciado otras formas de narrar, "otras" formas de decir, "otras" maneras de participar, y por eso me parecen muy potentes estos nuevos escenarios, teniendo siempre en mente que estamos inscritos en una lucha por el poder simbólico y por el poder político".

Juliana Jiménez: "Ampliando un poco lo que ya se ha dicho sobre el tema, estamos de acuerdo con que nos estamos moviendo en un escenario de prácticas cuando hablamos de ciudadanía digital, a mí me gustaría decir que estas prácticas son y no son las mismas, es decir, hablamos de prácticas sociales que tienen una misma naturaleza pero que han venido transformándose en gran medida por la aparición de diferentes tecnologías y la convergencia de lenguajes en el ciberespacio, aquí el papel de la comunicación o lo que vendría a ser el campo de la comunicación tiene que ver con encargarse de analizar estos cambios, justamente con el fin de proponer permanentes estrategias de adaptación a este ecosistema que es mutante, que todo el tiempo está transformándose y cada vez más velozmente; en esa medida pienso que la comunicación es la que justamente se encarga de analizar esos cambios para ir proponiendo estrategias de adaptación.

Aquí el asunto es cómo esas prácticas sociales, que no son nuevas, en tanto considero que son las mismas prácticas de siempre, para poner un ejemplo: de la lectura se espera un proceso de comprensión, un proceso de análisis, un proceso de crítica, esto no cambia, la lectura sigue siendo la misma en ese sentido, sin embargo, hay unos elementos que son ajenos a la lectura que sí se transforman, y ahí es donde viene la pertinencia de la comunicación. Siguiendo con el ejemplo de la lectura, ¿qué es lo que cambia?, el acceso a los textos, las formas de los textos, los dispositivos, los formatos a través de los cuales nos ponemos en contacto con los textos, las posibilidades de tránsito entre los textos, el hipervínculo entre los textos, aquí entra la comunicación a ver de qué manera aporta para generar ese tipo de estrategias, ante esos cambios en nuestras prácticas cotidianas".

Harvey Murcia: "Como lo han expresado ya mis colegas, quisiera acotar el concepto que la profesora Juliana Jiménez ha venido trabajando, y es el de la ciudadanía vista como una práctica, y cómo desde esa práctica empiezan a comprenderse otras emergencias, otras rupturas. ¿De qué tipo?, de relaciones con el mundo, de relaciones con el otro, de relaciones consigo mismo y con el medio ambiente, entonces estas prácticas dentro de la comunicación nos permitirían comprender lo que bien ha enunciado el profesor Valderrama, que es la lucha por el sentido y las significaciones sobre el mundo, entonces, una pista para entender estas nuevas relaciones, es la forma como se está narrativizando la subjetividad en distintas plataformas y dispositivos, que 
no solo tienen que ver con el escenario de lo virtual, sino que también trascienden al escenario de la vida cotidiana y de esta manera empezar a comprender cuales son las formas de circulación, las formas de producción y consumo que se empiezan a dar en estas ciudadanías, y que rebosan y ponen en crisis esas prácticas con las que veníamos construyendo la vida cotidiana".

Miguel Ezequiel Badillo Mendoza: "Una perspectiva desde la cual he encontrado un análisis muy interesante es la de ver la ciudadanía como un elemento discursivo, no solamente como una práctica exclusivamente política de la democracia representativa. Desde allí hay una línea muy atrayente, y es el hecho de que tengamos nuevas ciudadanías y toda esta serie de espacios que están surgiendo. Hay un gran error cuando se aduce, o se considera que significa un funcionamiento mejor; que Internet nos garantizaría una mayor participación, o que el tener todos acceso a determinados recursos permitirá un ejercicio de la política de una y otra forma, como tampoco garantiza que los grupos o movimientos sociales que tengan acceso a Internet tengan una mayor cualificación en sus procesos. Allí radica la complejidad de estas nuevas dinámicas. Desde allí me parece fundamental que la comunicación esté permitiendo la conexión y la construcción de redes, uno de los elementos en los cuales se soportan estas nuevas ciudadanías, lo cual no significa que tener acceso a ese recurso ya esté garantizando una mejor comunicación.

Considero que para nosotros los comunicadores estos escenarios abren unas diásporas y exigen una perspectiva más ecológica, esa visión de que unos medios sí y otros medios no, que debía ser de una forma o de la otra, o un enfoque de la comunicación u otro se rompe y reclama una comprensión más amplia. Y frente lo que es una ciudadanía digital, la comunicación debe constituir, validar y permitir recuperar esa capacidad de deliberación, que es lo que en determinadas instancias se pierde en estas nuevas esferas que se están generando".

Harvey Murcia: “Es más una apreciación de parte mía, creo que hay dos instancias que planteas que es importante resaltar en este momento, lo primero es que yo creo que cuando se habla de la práctica, la práctica misma es un discurso, lo discursivo es una práctica social, como bien lo recuerda Eliseo Verón, si podemos entender las prácticas que se están dando desde lo discursivo, que se dan por la lucha del poder y la lucha del sentido, la comunicación debe empezar a abordar esos fenómenos para entender cuáles son esas nuevas narrativas y esas nuevas ideas del mundo que están circulando, cómo se están consumiendo y cómo se están apropiando.

Y en una segunda instancia, me parece relevante lo que estás diciendo de empezar a comprender la diáspora en este nuevo ecosistema, justamente porque nos permitirá entender los efectos que están teniendo los medios sobre las subjetividades o sobre las corporalidades, y en una segunda instancia, los efectos sobre las dimensiones cognitivas de estas subjetividades, para entender las tensiones, las resistencias y lo que realmente está en disputa".

Carlos Valderrama Higuera: "Insisto en algo que está planteando el profesor Harvey sobre esa emergencia de nuevas subjetividades en el ejercicio de lo político, y en lo que acaba de plantear, en relación con la incidencia de ese escenario tecnológico y comunicativo en los cuerpos y en las subjetividades, porque me parece que uno de los componentes claves en de esas transformaciones de la ciudadanía y que tienen muy buena expresión en la mediación que hacen las tecnologías digitales de la información y la comunicación, es la presencia cada vez más creciente o por lo menos más evidente de lo estético como una parte de la dimensión de la política. Con lo estético no solamente me refiero al arte, o al uso instrumental del arte, más desde la perspectiva que lo plantea Ranciere, lo estético como aquello que aborda la distribución de lo sensible, por allí pasa el reconocimiento del otro, por allí pasa la dialogicidad, la capacidad de argumentar, de reconocer las otras culturas, de reconocer la diferencia, de jerarquizar lo estético y lo no estético, o des-jerarquizar en procesos de resistencias frente a ciertas maneras de entender subjetivamente y sensiblemente el mundo.

Y me parece que ese es uno de los rasgos claves que hoy se están presentando, que en buena parte atribuyo no de forma determinista, pero si de un modo en que las tecnologías digitales de la información y la comunicación y ese ecosistema digital permiten la emergencia de esas subjetividades a las que el profesor Harvey aludía".

\section{¿Desde su área de desempeño cómo lo evidencia?}

Camila Pérez Lagos: "En mi caso, me interesan particularmente las cuestiones relativas a la vigilancia digital y al derecho a la vida privada en la red, a mi juicio estos dos puntos hacen parte de las competencias que un ciudadano digital debe desarrollar. La vigilancia numérica es un tema que la ficción ha tratado ampliamente, esta mañana citaban a Orwell, más recientemente vemos series en Netflix como Black Mirror, Dark Net y Follow This que tratan esencialmente el tema de las tecnologías de Internet como un universo un distópico; sin embargo, las series muestran algo que nos ocurre más cotidianamente de lo que quisiéramos. Por ejemplo, podemos citar el uso del algoritmo "Compas", que es una herramienta que se usó en diferentes Estados de Estados Unidos y que permite "ayudar" a los jueces a tomar decisiones al momento de pronunciar una sentencia. Este algoritmo permitiría predecir la criminalidad de un individuo sobre la base de parámetros que solo 
el dueño de la herramienta conoce. Haciendo eco a la conversación de esta mañana, este es un nuevo ejemplo del secretismo con el que funcionan estos mecanismos. En enero de este año el algoritmo fue acusado de tener un sesgo racista ya que arrojaba más falsos positivos a ciudadanos que eran afrodescendientes.

Otro ejemplo es el uso de programas de "seguridad" que registran el comportamiento de un conductor con el fin de enviar los datos a las aseguradoras de automóviles y proponer seguros "adaptados". 0 también, en función de la localización a través de tarjetas electrónicas, se ofrecen de seguros de viaje. Estos son algunos ejemplos que evidencian el registro constante e indiscriminado de datos en la web.

Sin embargo, la vigilancia digital traería consigo algunos problemas mayores, además de los evidentemente personales y económicos, para las democracias. García Canclini en una entrevista en 2017 plantea que vivimos en la ilusión de la participación dentro de una burbuja digital, donde compartimos nuestras opiniones con quienes están de acuerdo, esto reconfigura el "cerco mediático" digital. Junto con lo anterior, la vigilancia digital pone en peligro la libertad de expresión de los ciudadanos; por ejemplo, el documental Nothing to hide muestra cómo la población acepta la vigilancia indiscriminada desde la quimera de la seguridad.

Foucault, en 1975 en "Vigilar y castigar", demostró que cuando un individuo se siente observado el comportamiento tiende a ser más conformista y consensual, esto es lo que se conoce como un efecto paralizador, la inhibición del derecho legítimo a ejercer la libertad de expresión por miedo a las represalias. Un investigador norteamericano descubrió que la mayoría de los musulmanes estadounidenses cree que el gobierno estadounidense supervisa sus actividades después del 11 de septiembre en EE.UU. y esto en consecuencia ha cambiado la manera como ellos utilizan Internet y lo mismo ocurre con la visita a páginas como Wikipedia, así como las páginas sobre terrorismo o Estado Islámico, que han bajado sus visitas ampliamente.

Esto sería un miedo, al parecer justificado, ya que la vigilancia digital además de ser un fenómeno ubicuo es perfectamente legal. Por ejemplo, luego de los atentados terroristas de 2015 en Francia, la ley de espionaje sirvió para legalizar el uso de cajas negras en los operadores de Internet que permitían vigilar el tráfico e identificar los potenciales terroristas. Sin embargo, según el Consejo Nacional de lo Digital, el mecanismo seria ineficaz ya que el comportamiento del terrorista no presenta una frecuencia que permitiera automatizar su identificación, dando cabida a tantos falsos positivos que sería imposible hacer un verdadero reconocimiento de quiénes son terroristas y quiénes no.
En este contexto complejo, yo estoy trabajando en el análisis del reciente "escándalo Cambridge Analytica" que, a mi juicio, reveló una crisis de confianza hacia la industria numérica. Para los que no conocen, Cambridge Analytica es una empresa británica que utilizó datos privados provenientes de usuarios de Facebook para convencer a los indecisos de votar por Donald Trump y desanimar a los votantes de Hillary Clinton con noticias falsas y con desinformación. Si recuerdan, Mark Zuckerberg reconoció ante el parlamento europeo hace unos meses, haber usado los datos de 87 millones de usuarios de Facebook.

Este caso evidencia que la protección de la vida privada es un tema que debe ser tratado como un tema político y que solamente asegurar la vida privada de los usuarios garantizaría el buen funcionamiento de las democracias actuales.

Algunos intentos por subsanar esta carencia es la nueva ley europea sobre datos personales, una ley que fue puesta en marcha solo en mayo de este año y que según algunas organizaciones no gubernamentales, lo único que permite es visibilizar el consentimiento del usuario para el uso de sus datos.

Facebook ha implantado toda una propaganda para cliquear y permitir al usuario decidir qué cosas muestra y qué cosas no, sin embargo, el escándalo de Cambridge Analytica va en completa oposición con eso.

Otra respuesta de parte de la llamada clase política es la del líder del partido laborista británico, Jeremy Corbyn, que en 2011 elaboró un manifiesto llamado "El manifiesto por la democracia digital", hay muchos puntos interesantes, está el tema del acceso universal a internet y el hecho de que los ciudadanos puedan controlar sus datos personales y asegurar la protección del derecho a la vida privada. Para concluir, comparto, además de la necesidad de leyes que protejan la vida privada en la era digital, la necesidad de educar al ciudadano digital.

El ejercicio o la práctica de la ciudadanía digital no reduce al individuo al papel de elector, o de usuario, sino al de actor, miembro y participe de la sociedad que lo rodea, atento al entorno y al respeto de sus libertades y derechos".

Carlos Valderrama Higuera: "Voy a referirme a una de las líneas de trabajo de estos últimos años y que tiene que ver con el uso de las tecnologías digitales de la información y la comunicación por parte de los movimientos sociales, para evidenciar esto que hemos estado conversando. Un primer aspecto está relacionado con que algunos de los movimientos sociales y, específicamente, los movimientos más tradicionales, hablo de los sindicatos, siguen llevando al espacio digital las prácticas tradicionales; en 
una investigación que hice sobre internet y cuatro sindicatos en Colombia, realmente no encontré allí ninguna innovación en la práctica de comunicación política, su estructura, su modo gestionar los procesos de comunicación, los modos de usar la información, efectivamente seguían siendo de carácter tradicional, verticales, sin espacios para el dialogo, para la argumentación, para la construcción colaborativa, etc. Pero también en otras investigaciones más recientes y que forman parte de mi tesis doctoral, estoy trabajando con el movimiento campesino en Colombia, y encuentro otras prácticas de la comunicación política muy diferentes, muy distantes a esas prácticas tradicionales; ya se señalaba una de ellas que me parece muy importante en esos escenarios, el trabajo en red, porque allí se rompen las estructuras organizativas verticales, autoritarias, que fomentan la heteronomía, más bien lo que se construye y lo que se está gestionando desde el punto de vista político, pero también desde el punto de vista de la comunicación política y del ejercicio de la ciudadanías, es el trabajo colaborativo, la construcción de redes, redes que se enredan con otras redes, unas construcciones absolutamente complejas, flexibles y muy dinámicas. Eso conlleva otras maneras de generar y propiciar los debates, de elaborar unas estrategias de prevención y sobre todo en Colombia, de generar alertas tempranas frente a esas arremetidas en estos últimos años contra líderes sociales y defensores y defensoras de derechos humanos, que también permite gestionar de otros modos los procesos de la narración política y de construcción colectiva.

Desde mi experiencia lo que he visto es que justamente, aun desde el campo de los movimientos sociales, sin entrar a mirar la dinámica de la comunicación política de los partidos tradicionales, convergen distintos tipos de prácticas tradicionales que se trasladan y que navegan en la red, y también emergencias que hay en la red y que van a las prácticas y las dinámicas de lo presencial. Eso es lo que yo fundamentalmente tendría para decir frente a la tercera pregunta".

Juliana Jiménez Rodríguez: “Nos gustaría hablar, y digo nos gustaría, ya que estamos trabajando en equipo actualmente un proyecto de investigación. Nos gustaría hablar desde la perspectiva de la cotidianidad y retomar el tema de las habilidades, de las competencias y de esas prácticas que involucran al ser humano y lo hacen transitar en lo digital, como decía de manera parcial, y que le permite actuar e interactuar gracias a diferentes tecnologías. En el caso puntual, las prácticas sobre las cuales nos hemos interesado tienen que ver con la construcción de la memoria, más individual que a nivel colectivo, sin embargo, somos conscientes de que estas prácticas siempre van a estar permeadas por lo colectivo.

Nos interesa mucho mirar qué pasa en ese escenario que se ha mantenido limitado por una serie de espacios y de tiempos antes del ciberespacio, muy ligados a lo físico, a los objetos, qué pasa cuando estas prácticas se empiezan a construir desde la experiencia en lo digital, y no solamente en la experiencia, sino que también el recuerdo empieza a trabajarse desde el entorno digital. En este sentido, los individuos tienen mucho por reflexionar, por mirarse a sí mismos, de hecho, nosotros mismos nos preguntamos qué ha pasado con esas formas que hemos tenido de narrarnos, porque finalmente las prácticas de memoria tienen que ver con esas formas de contarnos y de resignificar lo que nos ha pasado y de seguir construyendo una historia basados en esas resignificaciones".

Harvey Murcia: "El interés se centra en tratar de comprender estas nuevas prácticas de memoria que se están dando en el escenario digital, entonces la evidencia empieza a aparecer como bien lo decía el profesor Valderrama, cómo empieza a haber una redistribución de lo sensible y esta distribución de lo sensible nos permitiría comprender cómo emerge una estética "otra", donde se defienden unas subjetividades, donde se defienden unas narrativas, unas relaciones con el otro, con el medio ambiente, con el espacio, con lo político, con lo ético, que van permitiendo comprender este recuerdo como bien lo decía el profesor Brean en alguno de sus textos, como una memoria RAM, donde cada vez más se pierde una relación directa con lo cotidiano, y empieza a aparecer una mediatización digital que reconfigura el mapa social y por lo tanto reconfigura lo cognitivo, allí es donde nosotros creemos que las ciudadanías digitales empiezan a emerger como un espacio de lucha y resistencia, pero al mismo tiempo, como una subordinación a ciertas hegemonías que están circulando. No podemos ver las cosas en blanco y negro, sino que también hay que verlas en escala de grises, para poder comprender en última instancia que cuando hablamos de ciudadanías digitales estamos hablando de una práctica muy compleja atravesada por un sin número de actividades, de ideas, de tecnologías que están haciendo y deshaciendo permanentemente este horizonte que está llegando a nosotros en la contemporaneidad".

Miguel Ezequiel Badillo Mendoza: "Frente al tema de cómo lo evidencio, voy a explorar tres escenarios, uno fue mi tesis doctoral, donde hice todo un rastreo con relación a un proyecto que se llama la Colosa, en el Tolima; un proyecto de minería a cielo abierto, en el que la AngloGold Ashanti empezó todo un ejercicio de exploración y la comunidad de una u otra forma empezó a hacer todo un movimiento de protesta y de denuncia. Se tenía un antecedente muy importante del proceso inicial de San Turban, allí las redes sociales empezaron a tener una dinámica muy fuerte, acá era otro contexto, mucho más rural, mucho más campesino. Se hizo un ejercicio inicial de exploración de uso de medios de comunicación digital, empezamos a encontrar que se generaba mucha información pero pudimos encontrar que solamente dos o tres experiencias tenían una dinámica mucho más completa; utilizamos unos modelos de análisis que en Europa se les llama contrainformación, acá en Latinoamérica puede ser el equivalente a la comunicación alternativa y encontramos dos experiencias, una que se llama "Conciencia Campesina", y otra que se 
llama Blog "No a la Colosa Horrorosa"; se hizo un análisis inicial de toda esa dinámica, y después empezamos a encontrar el concepto que hoy yo llamo ciberciudadanía, equivalente al concepto de ciudadanía digital. Encontramos, que si bien allí había un componente político muy importante, fue clave que a medida que el conflicto fue avanzando, (estamos hablando de un conflicto de casi doce años), las organizaciones también evolucionaron en su accionar; empezaron a detectarse una serie de herramientas jurídicas, que fueron las consultas populares, que empezaron a mostrar unos escenarios de acción colectiva, por lo cual, la propia dinámica de los movimientos sociales se centró en eso. Desde el inicio, los movimientos buscaron cómo bloquear el proyecto minero y lo lograron con las consultas populares, la primera fue en el municipio de Piedras, donde se detienen los procesos de exploración y explotación; a tal punto se instaura, que en 2018 casi que el proyecto de locomotora minera del gobierno Santos quedó bloqueado, porque las consultas populares establecen que la ciudadanía tiene que ser consultada entre comillas, si quiere que ese tipo de proyectos se lleven a cabo en su territorio.

Lo que empezaron a hacer estas organizaciones, fue ubicar un contexto de política situada como hablaba, directamente con elementos jurídicos aprobados por la constitución pero a la vez esto iba acompañado de una movilización digital muy importante. Se fueron mejorando y cualificando las formas de comunicar, las formas de conectar, aprender a usar las herramientas digitales, básicamente Twitter y Facebook, empezaron a generarse elementos estéticos, aparecieron avisos contra informativos supremamente ofensivos, allí no había mediación con el lenguaje, eran casi insultos. Otro aspecto muy interesante era el elemento cultural, había unos elementos de apropiación de lo ancestral, lo terrenal, lo rural, que le daba mucha fuerza a ese concepto de ciudadanía.

Otro referente a tener en cuenta es que cuando hablamos de ciudadanías digitales vamos al elemento político, pero vale la pena correr ese horizonte y las fronteras hacia lo que pasa en el día a día. Estoy muy de acuerdo con lo que dice Juliana, ¿qué pasa con ese sujeto que está en su interacción diaria con las demás personas?, madres que la forma de saber dónde está el hijo es través de Facebook y ahora es con WhatsApp, creo que allí también hay un elemento de ciudadanía digital donde no está el elemento de lo participativo y lo democrático, sino más ese sujeto que está en el devenir de su existencia en unos entornos, como el ciberespacio, donde muchas cosas suceden y casi que la gente se va insertando sin preguntarse qué pasara allá, con todo lo positivo y negativo, con todos los riesgos y no riesgos a los cuales se enfrenta una persona en dichos escenarios.

Y finalmente, lo percibo mucho en el tema de la investigación, en este momento algo que nos interesa en el grupo de trabajo es el tema de las metodologías que estamos utilizando para investigar las redes digitales, a veces estamos haciendo un ejercicio de traer de otros escenarios métodos para analizar lo que está pasando en estos sistemas digitales y en estas ecologías digitales, ahí es donde yo percibo que la academia se está insertando y requerimos también pensarlo, otras áreas ya lo tienen más claro, por ejemplo el mercadeo, la publicidad, ellos si tienen muy preciso qué necesitan y qué buscan en este tipo de escenarios, creo que nosotros todavía estamos en indagaciones frente a ello, esas son mis perspectivas.

Los invito compañeros, si de pronto hay algún comentario adicional a lo que estamos aquí agregando, si no, aquí hay dos preguntas que nos han formulado, me gustaría traerlas al escenario y ver desde allí que podríamos agregar o quien podría responderlas.

Básicamente son dos preguntas de una persona en Facebook, ¿Cómo aprovechar lo digital sin perder lo esencial de la comunicación, como lo tradicional? y la segunda ¿si se dice que todos tenemos acceso a las redes sociales, por qué no todos interactúan y solo reciben información?"

Carlos Valderrama Higuera: "Quiero referirme a la segunda pregunta, creo que la formación del sujeto moderno y de la subjetividad moderna en general y por supuesto las subjetividades políticas en particular, desde el proyecto moderno, son subjetividades heterónomas, es decir el sujeto que construye la modernidad es un sujeto heterónomo, a nosotros nos han educado en la heteronomía y no en la autonomía, y por eso es muy difícil salirnos de ese esquema, empoderarnos, es muy difícil ser productores de información, productores de ideas y de propuestas, de hecho, el mismo sistema de la democracia representativa parte de esa constitución de un sujeto heterónomo que delega su participación política en otro. Es un sistema delegatario, el sistema representativo de la democracia que prima en el escenario actual de la sociedad, no basta con tener acceso, no basta con acceder a la web 2.0, y a todas las tecnologías, no basta tampoco con desarrollar unas competencias instrumentales para saber usar las distintas aplicaciones, y las distintas técnicas de esa gran tecnología de la información digital, eso pasa por la reconstitución de las subjetividades a las que hemos estado aludiendo permanentemente en esta conversación, y nos plantea un reto en todos los escenarios, en el de la academia en particular y es por la formación de ciudadanos, aunque yo preferiría hablar mejor de sujetos y subjetividades políticas, con lo cual vale preguntarnos ¿qué estamos haciendo nosotros ahí, como universidad en ese escenario de la actualidad?"

Miguel Ezequiel Badillo Mendoza: “Carlos muchas gracias, yo invitaría a todos los participantes a que tratáramos de condensar, cada uno en unas pocas palabras una especie de idea fuerza, puede ser conclusión, recomendación, comentario; aquí hemos trazado unos derroteros importantes, han quedado unas líneas claves para entender lo que es la 
ciudadanía digital, entonces los invitaría para cerrar, a que cada uno pueda sintetizar en una idea fuerza lo que queda de estas casi dos horas de conversatorio".

Camila Pérez Lagos: "Mi intervención llevaba un poco a la pregunta de Luis Monsalve, que es el tema de la ética y la ciudadanía digital, y cómo los ciudadanos también están protegidos en su actuar. Los sindicatos en Francia fueron muy reacios a utilizar las redes sociales, justamente por este miedo, por esa mirada apocalíptica de las redes. Las grandes empresas que monopolizan Internet, las GAFAM, no pueden asegurar la ética, para mí son los Estados. Europa ha sido precursor en ello, pero por otro lado también son los usuarios, hay que crear esas competencias de participación, es lo mismo que ocurre cuando un ciudadano elije no ir a votar, no por una decisión racional, sino porque no se siente interpelado, no siente que eso afecte su vida cotidiana, lo mismo ocurre con la participación en redes sociales, por lo mismo me interesó el cambio que, a mi juicio, hubo después del "escándalo Cambridge Analytica". En un análisis que hicimos de cómo se desarrolló este escándalo en Twitter, observamos que los usuarios se apropiaban del tema de la privacidad digital, por ejemplo, la gente se aconsejaba, encontraban maneras de hacer búsquedas en Internet de forma privada, entonces surgió una responsabilidad individual frente a los Estados que tenían leyes arcaicas y a las GAFAM que ejercían prácticas poco éticas".

Carlos Valderrama Higuera: “Creo que hay una aclaración de Esperanza Peña con relación a la primera pregunta, dice que si cuando hablamos de tecnología o ciberespacio ya salimos de lo tradicional. Creo que no. Hay una confusión, lo tradicional no está dado por el uso o no de ciertas tecnologías, lo tradicional en el ejercicio de la ciudadanía y en el ejercicio de lo político, alude más bien a los escenarios tradicionales de la acción política, es decir aquella acción institucional, de los partidos, de los parlamentos, del Senado y a un ejercicio de la democracia representativa, y no necesariamente a la mediación tecnológica. Así con lo emergente, aludiríamos al alejamiento de ese ejercicio político tradicional y a esa ciudadanía circunscrita a derechos y deberes, y más bien a lo que Chantal Mouffe ha llamado el ejercicio de lo político por tensión con la política, y en esa medida entonces aprovechar lo digital sin perder lo esencial de la comunicación no aludiría a eso, porque no existe lo esencial de la comunicación, la comunicación es conflicto, es construcción de sentido, es lucha, es debate, es ahí donde se podría aprovechar efectivamente la potencia de lo digital para ampliar ese escenario de lo político, del debate, de la lucha política, del despliegue de esas nuevas subjetividades, de eso otro ético y estético que hoy atraviesa a los nuevos ejercicios de la ciudadanía, aquello que insisto han planteado varios autores, como es el hecho de qué aspectos de la cultura, cuáles aspectos de la vida privada, de la vida íntima, devienen en hechos políticos hoy".
Juliana Jiménez Rodríguez: “Quisiera recalcar el tema de la adaptación, y está relacionado con la pregunta que hacía el participante a través del chat de Facebook Live y es cómo los constantes cambios o las constantes transformaciones en el ecosistema educativo nos exigen justamente eso, un proceso, una adaptación. Pero hay una realidad, creo que es casi una verdad, algo que hay que asimilar, y es que las diferentes habilidades o capacidades relacionadas con la comunicación vienen cambiando desde el inicio de los tiempos, a medida que avanzan o aparecen diferentes tecnologías, esto no es algo nuevo, a propósito de internet o el ciberespacio, esto es algo que ya lo hablaba Platón, y ya desde la aparición de la escritura había un temor a perder la oratoria, es lo que nos viene pasando a los seres humanos frente a los procesos comunicativos desde siempre, es uno de los principios para dejar de satanizar este tema y ver más bien que esas prácticas siguen siendo en parte las mismas y en parte cambiantes, el hecho de tener un Smartphone hace que no tengamos la misma capacidad de memoria que teníamos los seres humanos hace diez o veinte años, eso no es algo que tengamos que detener o combatir, sino que hace parte de todo ese proceso de adaptación, esa sería una de las ideas con la que quisiera terminar".

Harvey Murcia: "Para finalizar, estamos frente a un nuevo paradigma que nos está invitando a pensar y repensar los cánones y las tramas de la cultura, y esto es lo que debemos tener presente para adentrarnos en esta aventura que significa comprender las nuevas formas sociales, hacernos preguntas sobre la relación de la política y la comunicación, la cultura y la comunicación, subjetividades, narrativas, y narrativizaciones de la vida contemporánea".

Miguel Ezequiel Badillo Mendoza: "Un texto que trabajé decía que el reto de la comunicación en este momento era lograr comprender estas cosas, porque sin la comunicación no estaríamos hablando de ello; cuando hablamos de lo digital o del concepto de ciudadanía en todas estas dinámicas, comprendidas como elementos discursivos, comprendidas como prácticas sociales, hay un elemento transversal que es la comunicación, el pensar un ciudadano digital es un reto para nosotros como formadores de comunicadores sociales, que miremos esto más allá de que la persona sepa manejar Twitter, una red social, un dispositivo, que la perspectiva de la comunicación siempre va más allá de los medios, sea la radio, la televisión, ahora lo que se viene con los sistemas de comunicación digital, para mí se conserva, para que se comprenda que el periodista es algo más que una serie de herramientas que le permitan hacer su trabajo mucho más ágil, mucho más práctico, porque eso no se traduce en que las cosas estén bien o mal hechas, para mí es un ejercicio de la comunicación, de formar a esas personas que puedan comprender ese concepto de ciudadanía digital, pero también el pensar que a través de las tecnologías se movilizan una cantidad de contenidos que a mi juicio sobrepasan lo que 
como sujetos somos, la relación tecnologías-sujetos, sociedad, no va a la par, y cada vez las posibilidades que tienen las personas de producir y consumir información va a un nivel y un ritmo muy distinto, más en nuestros contextos donde hay tantas situaciones que lo demarcan, alfabetización, acceso, etc. Entonces yo pensaría que allí es importante tener ese punto de referencia".

"Bueno, estimados participantes, profesora Camila Pérez Lagos, profesor Carlos Eduardo Valderrama, Juliana Jiménez y Harvey Murcia, este es un primero punto, no va a ser el único, es una motivación del Grupo de Trabajo "Comunicación digital, redes y procesos" por empezar a dar estas posibilidades, ya que estamos hablando de lo digital, este tipo de dispositivos que tenemos como universidad permite que personas que estamos ubicadas en diferentes latitudes podamos tener este diálogo, y la idea es ir integrando nuevos colegas que estén motivados en estos temas". 